\title{
Chemokine Signaling Guides Axons within the Retina in Zebrafish
}

\author{
Qin Li, ${ }^{1}$ Komei Shirabe, ${ }^{1,2}$ Christine Thisse, ${ }^{3}$ Bernard Thisse, ${ }^{3}$ Hitoshi Okamoto, ${ }^{4}$ Ichiro Masai, ${ }^{5}$ and John Y. Kuwada ${ }^{1}$ \\ ${ }^{1}$ Department of Molecular, Cellular, and Developmental Biology, University of Michigan, Ann Arbor, Michigan 48109-1048, ${ }^{2}$ Department of Anatomy, \\ Biology, and Medicine, Oita Medical University, Hasama-machi, Oita 879-5593, Japan, ${ }^{3}$ Institut de Genetique et de Biologie Moleculaire et Cellulaire, 67404 \\ Illkirch, France, and ${ }^{4}$ Laboratory for Developmental Gene Regulation and ${ }^{5}$ Masai Initiative Research Unit, The Institute of Physical and Chemical Research \\ (RIKEN), Wako-shi, Saitama 351-0198, Japan
}

Chemokines are a large family of secreted proteins that play an important role in the migration of leukocytes during hematopoiesis and inflammation. Chemokines and their receptors are also widely distributed in the CNS. Although recent investigations are beginning to elucidate chemokine function within the CNS, relatively little is known about the CNS function of this important class of molecules. To better appreciate the CNS function of chemokines, the role of signaling by stromal cell-derived factor-1 (SDF-1) through its receptor, chemokine (CXC motif) receptor 4 (CXCR4), was analyzed in zebrafish embryos. The SDF-1/CXCR4 expression pattern suggested that SDF-1/CXCR4 signaling was important for guiding retinal ganglion cell axons within the retina to the optic stalk to exit the retina. Antisense knockdown of the ligand and/or receptor and a genetic CXCR4 mutation both induced retinal axons to follow aberrant pathways within the retina. Furthermore, retinal axons deviated from their normal pathway and extended to cells ectopically expressing SDF-1 within the retina. These data suggest that chemokine signaling is both necessary and sufficient for directing retinal growth cones within the retina.

Key words: zebrafish; retinal ganglion cell; SDF-1; CXCR4; axonogenesis; axon

\section{Introduction}

Chemokines are a large family of small, secreted proteins with important roles in the migration of leukocytes during inflammatory responses and in a variety of other processes, including development of the hematopoietic, lymphopoietic, and vascular systems (Rossi and Zlotnik, 2000; Tran and Miller, 2003) and migration by primordial germ cells (Doitsidou et al., 2002; Knaut et al., 2003) and lateral line sensory cells (David et al., 2002). A family of G-protein-coupled, seven-transmembrane receptors mediates chemokine action. Interestingly, chemokines and their receptors are expressed widely within the CNS (Cho and Miller, 2002) and despite their well-known role as chemotactic agents within the immune system, their function within the CNS is not well understood. Three known CNS targets of chemokines are the cells of the external granule layer in the cerebellum, granule cells in the dentate gyrus in the hippocampus, and retinal ganglion cells (RGCs). The chemokine, stromal cell-derived factor-1 (SDF-1), acts via the chemokine (CXC motif) receptor 4 (CXCR4) to mediate chemoattraction for migration by cerebellar and dentate granule cells. Interestingly, SDF-1/CXCR4 signaling can also regulate turning by cerebellar granule growth cones in

Received 0ct. 21, 2004; revised Dec. 29, 2004; accepted Dec. 30, 2004

This work was supported by National Institutes of Health Grants RR15402 (C.T., B.T.) and NS36587 (J.Y.K.). We thank Dr. H. Knaut for providing ody embryos and F. El Saeidi and S. Sprague for fish care.

Correspondence should be addressed to Dr. J. Y. Kuwada, Department of Molecular, Cellular, and Developmental Biology, University of Michigan, Ann Arbor, Ml 48109-1048. E-mail: kuwada@umich.edu.

DOI:10.1523/JNEUROSCI.4393-04.2005

Copyright $\odot 2005$ Society for Neuroscience $\quad 0270-6474 / 05 / 251711-07 \$ 15.00 / 0$ vitro (Xiang et al., 2002), can reduce repulsion of RGC growth cones by Slit 2 in vitro, and is required for proper pathfinding by the central axons of cutaneous sensory neurons (Chalasani et al., 2003a). These results suggest that SDF-1/CXCR4 signaling may mediate guidance of growth cones and perhaps other aspects of differentiation by neurons in vivo.

This report investigates the in vivo function of SDF-1/CXCR4 signaling for guidance of RGC axons in zebrafish embryos by using both gain- and loss-of-function strategies. Our results suggest that SDF-1/CXCR4 signaling plays an important role in the guidance of RGC growth cones to the exit site of the retina to the optic stalk.

\section{Materials and Methods}

Fish breeding and maintenance. Zebrafish were maintained as described previously (Westerfield, 1995), and embryos were staged by hour postfertilization (hpf) (Kimmel et al., 1995). Wild-type, ath5:gfp (Masai et al., 2003), hsp70:gfp, and $h s p 70: s d f-1 b-g f p$ were used in this study.

Cloning of zebrafish sdf-1b, sdf-1a, and cxcr $4 b$. A $1.4 \mathrm{~kb}$ fragment of the $s d f-1 b$ cDNA was identified from a cDNA expression screen (C. Thisse and B. Thisse, unpublished observation). A full-length cDNA $(2.9 \mathrm{~kb})$ was cloned by using the fragment to screen a 32-36 hpf zebrafish $\lambda$ gt 11 cDNA library (gift from K. Zinn, California Institute of Technology, Pasadena, CA). Sequence analysis revealed that the cDNA encoded a zebrafish SDF-1 homolog and was named $s d f-1 b$. A BLAST search using the $s d f-1 b$ sequence identified the zebrafish expressed sequence tag (EST) fj88h12 (GenBank accession number AW420224) that encoded another full-length SDF-1 homolog that was named sdf-1a. A basic local alignment search tool (BLAST) search using the mouse CXCR4 sequence identified the zebrafish EST fd10g03 (GenBank accession number 
AI959485) that contained the full-length $c x c r 4 b$ cDNA described previously (Chong et al., 2001).

Generation of expression constructs. Construct $p H s p 70 / 4-s d f-1 b$ was made by inserting the $s d f-1 b$ cDNA into the $p H s p$ 70/4-egfp vector (Halloran et al., 2000) to replace the enhanced green fluorescent protein (EGFP) coding sequence. Construct $p H s p 70 / 4-s d f-1 b$-egfp was made by amplifying the $s d f-1 b 5^{\prime}$ untranslated region (UTR) and coding region by PCR using primers that removed the stop codon and inserted into $p H s p 70 / 4-e g f p$. Construct $p H s p 70 / 4$ $s d f-1 a-e g f p$ was made by amplifying the $s d f-1 a$ coding region from EST clone fj88h 12 by PCR, cutting out the PCR product and inserting it into $p E G F P$ immediately upstream to the egfp coding sequences. The coding sequence of SDF-

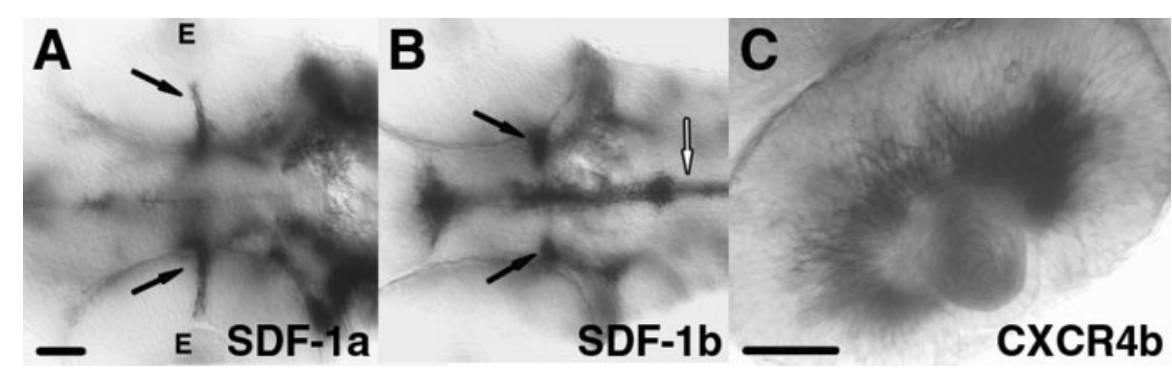
1A-GFP fusion protein was then cut out and inserted into $p H s p 70 / 4-e g f p$ to replace egfp. Construct $p H s p 70 / 4-c x c r 4 b$ egfp was made by amplifying the $c x c r 4 b$ coding sequence from EST clone fd10g03 by PCR, digesting the PCR product with EcoRI/BamHI, and cloning into $p E G F P$. The $c x c r 4 b-g f p$ coding sequence was then cut out and inserted into $p H s p 70 / 4-e g f p$ to generate the construct.

Generation of hsp70:sdf-1b transgenic fish and induction of SDF-1b. Transgenic fish were generated as described previously (Xiao et al., 2003; Li et al., 2004), except that the presence of the transgene was assayed by PCR using primers from the $H s p 70 / 4$ promoter and the $s d f-1 b$ coding sequence. Three hsp70:sdf-1b-gfp lines ( $h s p 70: s d f-1 b-g f p^{k 1-3}$ ) and two $h s p 70: s d f-1 b-3^{\prime}$ utr lines (hsp70:sdf-1b-3' utr ${ }^{k 4-5}$ ) that were heat inducible were established. The three $h s p 70: s d f-1 b-g f p$ lines varied in intensity of GFP fluorescence. For all experiments, $h s p 70: s d f-1 b-g f p^{k 1}$ embryos that expressed the highest intensity of GFP fluorescence were used. Control hsp70:gfp ${ }^{k 6}$ embryos were generated previously (Halloran et al., 2000). Embryos were heat induced as described previously (Xiao et al., 2003).

In situ hybridization, immunocytochemistry, and retinal cryosection. In situ hybridization and antibody labeling to whole-mounted embryos was performed as described previously (Schulte-Merker et al., 1992; Westerfield, 1995). The following antibodies were used for immunocytochemistry: anti-GFP (1:25,000); monoclonal antibody (MAb) Zn5 (1:500); anti-acetylated $\alpha$-tubulin (1:1000). Ten micrometer transverse sections of the retina were prepared as described previously (Westerfield, 1995).

Morpholino knockdown. Morpholino oligonucleotides (MOs) with $0.1 \%$ phenol red were injected into 1 - to 4 -cell stage embryos. The following antisense MOs were used to inhibit the translation of $s d f-1 a$ or $c x c r 4 b$ mRNA. The CAT sequence corresponds to the start ATG: antisense-sdf-1a MO, 5'-ACTTTGAGATCCATGTTTGCAGTG-3'; antisense-cxcr $4 b$ MO, 5' -AAATGATGCTATCGTAAAATTCCAT-3'.

In control experiments, standard randomized $\mathrm{MO}$ and a 4-base mispaired control MO for $c x c r 4 b$ ( $5^{\prime}$-AA $t$ TGA $a$ GCTATCGTAA $t$ ATTgCAT$\left.3^{\prime}\right)$ were injected. Approximately $2 \mathrm{ng}$ of the mixed antisense $s d f-1 a$ and $c x c r 4 b$ MO (1 ng each) was injected into each embryo. The amount of MO injected was estimated by the volume of the injected solution as denoted by the phenol red.

Generation of transgenic/wild-type mosaic embryos and ectopic expression of SDF-1b-GFP. Cell transplantation from $h s p 70: s d f-1 b$-gfp to wildtype embryos was conducted as described previously (Ho and Kane, 1990). The micropipettes for transplantation were pulled using thinwalled glass tube ( $1 \mathrm{~mm}$ outer diameter) with a Sutter microelectrode puller. The tips were broken to $30-40 \mu \mathrm{m}$ in diameter and then polished with a microforge. To better visualize transplanted cells in mosaic embryos, donor embryos were injected at 1- to 4-cell stage with fluorescent lineage tracer Alexa Fluor488 dextran (molecular weight, 10,000; Molecular Probes, Eugene, OR). Embryos were kept in embryo medium (Westerfield, 1995) at $28.5^{\circ} \mathrm{C}$ until the dome stage ( $4-5 \mathrm{hpf}$ ), and the chorions were removed manually. Embryos were mounted in a $3 \%$ methyl cellulose solution. The apparatus for transplantation was described by Westerfield (1995). Approximately 30-50 cells were injected into each host embryo near animal pole. After transplantation, mosaic

embryos were raised in embryo medium and heat induced by placing embryos in a Petri dish into a water bath at $37^{\circ} \mathrm{C}$ at 28,32 , and $36 \mathrm{hpf}$ for $0.5 \mathrm{~h}$ each and fixed at $40 \mathrm{hpf}$. Retinal axons were labeled with antiacetylated $\alpha$-tubulin, and donor cells were detected by GFP fluorescence or the fluorescent lineage tracer by confocal microscopy.

\section{Results}

\section{Expression of SDF-1 and CXCR4 correlate with retinal axon outgrowth}

In zebrafish, there are two cDNAs, $s d f-1 a$ and $s d f-1 b$, that appear to be equally homologous to mammalian $s d f-1$. $s d f-1 b$ was identified from sequence and expression analysis of random zebrafish cDNAs and $s d f-1 a$ from an EST identified from a BLAST search. The two cDNAs excluding the sequences encoding the signal peptides show 91\% similarity with SDF-1a, 63\% similarity with human SDF-1, and 64\% similarity with SDF-1b. In zebrafish there also are two SDF-1 receptors, CXCR4a and CXCR4b, that are highly homologous to each other and equally homologous to mammalian CXCR4, suggesting that the CXCR4 gene was duplicated in the zebrafish genome (Chong et al., 2001). The high level of homology between SDF-1a and SDF-1b and the equal homology between them and human SDF-1 suggests that SDF-1a and SDF-1b are duplicates much like many other genes in teleost genomes (Postlethwait et al., 2000).

The two $s d f-1$ genes are expressed in specific and dynamic patterns with little overlap ( $\mathrm{Li}$ et al., 2004). One place where both $s d f-1 \mathrm{~s}$ are expressed is by cells in the portion of the optic stalk that is adjacent to the eye (Fig. $1 A, B$ ). In the optic stalk, sdf-1a expression extends right up to the retina, whereas $s d f-1 b$ is expressed more distally from the retina. Embryos hybridized with an $s d f-1 a$ riboprobe and their retinal ganglion cell axons labeled with antiacetylated $\alpha$-tubulin showed that the axons extend along cells expressing $s d f-1 a$ (data not shown). Furthermore, $c x c r 4 b$ but not cxcr $4 a$ is expressed by cells that, by their position, are likely to be retinal ganglion cells during the period of their axonogenesis (Fig. 1C) (Chong et al., 2001). Thus, the expression of the two chemokines and their putative receptor suggest that SDF-1 secreted from the optic stalk attracts the axons of CXCR 4 bearing RGCs to the exit site of the retina into the optic stalk.

\section{Antisense knockdown of SDF1/CXCR4 signaling leads to pathfinding errors by retinal ganglion axons}

Because the SDF-1s are well situated to guide RGC axons to the exit site of the retina, we wondered whether interference with SDF-1/CXCR4 signaling might cause RGC axons to follow aberrant pathways within the retina. To determine whether SDF-1/ CXCR4 signaling is required for guidance of RGC axons, we in- 

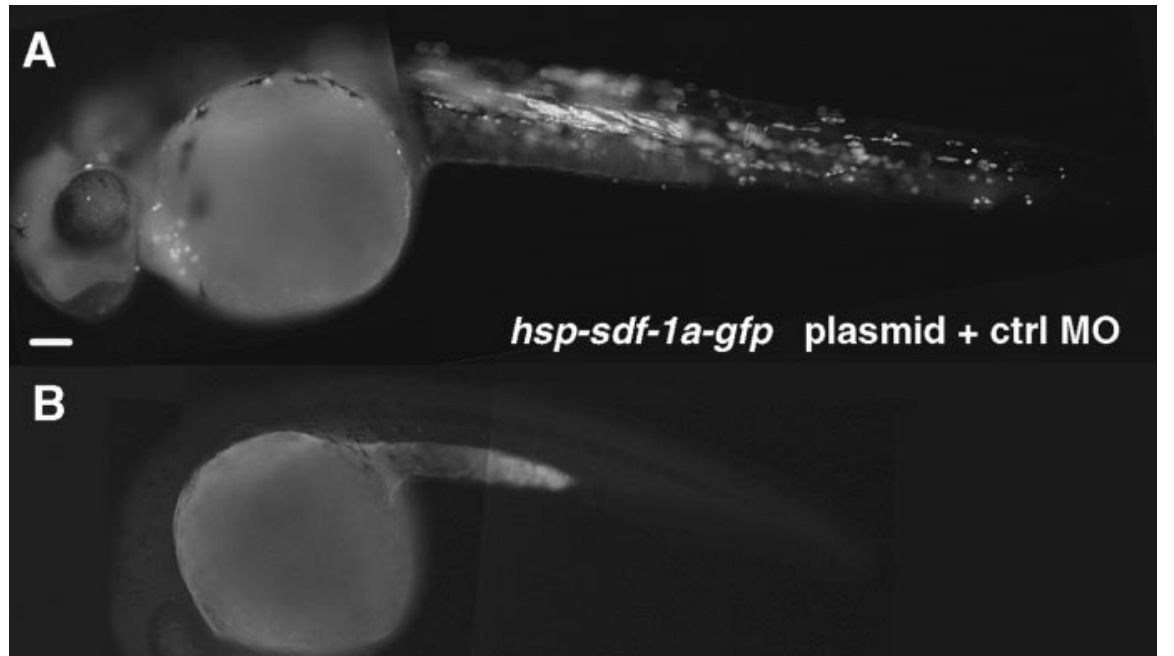

hsp-sdf-1a-gfp plasmid + sdf-1a MO

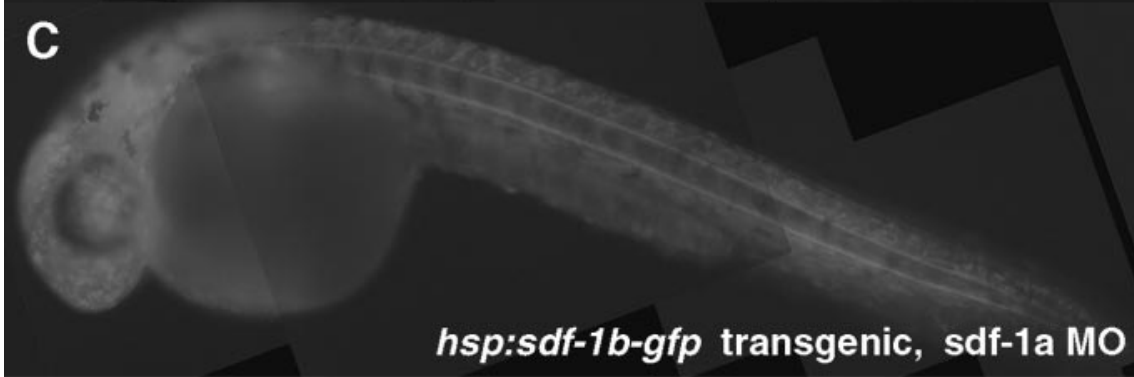

Figure 2. M0 against sdf-1a efficiently and specifically knocks down the synthesis of SDF-1a. All panels show 28 hpf embryos that were heat induced at $24 \mathrm{hpf}$. A, Wild-type embryo coinjected with hsp70-sdf-1a-gfp expression construct and standard control morpholino, showing mosaic expression of SDF-1a GFP after heat induction. B, Coinjection of hsp70-sdf-1a-gfp plasmid and antisense sdf-1a $\mathrm{MO}$ effectively blocked protein synthesis of targeted transcript, as indicated by the absence or severe reduction of GFP fluorescence. Because the heat-inducible expression of hsp70-sdf-1a-gfp construct is much higher than the endogenous expression of sdf-1a, similar dosage of antisense sdf-1a $\mathrm{MO}$ can presumably effectively knock down endogenous sdf-1a expression as well. C, A heatinduced $h s p 70: s d f-1 b$-gfp transgenic embryo injected with antisense sdf-1a morpholino, showing induced, ubiquitous expression of SDF-1b GFP, of which expression was not affected by antisense sdf-1a M0. Scale bar, $40 \mu \mathrm{m}$.

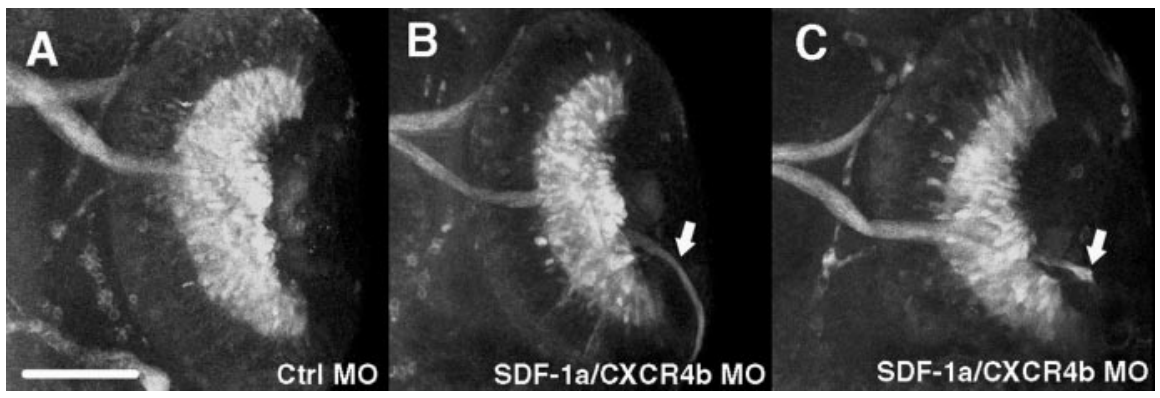

Figure 3. Knockdown of SDF-1a and CXCR4b led to pathfinding errors by RGC axons. All panels are ventral views of retinas of 48 hpf ath 5:gfp transgenic embryos. A, The optic nerve and RGCs marked by GFP fluorescence in a 48 hpf ath5: gfp transgenic embryo injected with $2 \mathrm{ng}$ of control MO. B, C, Injection of $1 \mathrm{ng}$ each of $s d f-1 a$ and $c x c r 4 b$ antisense MOs leads to pathfinding errors by RGC axons (arrows). Scale bar, $20 \mu \mathrm{m}$.

jected antisense MOs (Nasevicius and Ekker, 2000) against $s d f-1 a$ and $c x c r 4 b$ into recently fertilized embryos to knock down the encoded proteins. To test whether $s d f-1 a$ and $c x c r 4 b$ antisense MOs were effective in knocking down, respectively, SDF-1a and CXCR4b translation, we injected the antisense $\mathrm{MO}(\sim 3 \mathrm{ng})$ at the 1 - to 4-cell stage followed by injection of the heat-inducible expression construct for $s d f-1 a$ or $c x c r 4 b$ at the 8-32 cell stage. In each case, the antisense MO effectively knocked down the target protein, whereas the control MO did not ( $n=90$ for SDF-1a) (Fig. 2$)(n=61$ for CXCR4b) (data not shown). To examine the specificity of the MOs, we injected $s d f-1 a$ antisense MO ( $\sim 3 \mathrm{ng})$ into recently fertilized hsp70:sdf-1b-gfp embryos $(n=29)$. The antisense MOs did not suppress heat induction of GFP fluorescence (data not shown). These results demonstrate that SDF-1a and CXCR4b can be effectively and specifically knocked down by injections of the appropriate antisense MO.

We examined the effects of SDF-1a plus CXCR4b knockdown by injecting antisense $s d f-1 a$ plus $c x c r 4 b$ MOs $(\sim 1 \mathrm{ng}$ of $s d f-1 a \mathrm{MO}$ and $1 \mathrm{ng}$ of $\operatorname{cxcr} 4 b \mathrm{MO}$ ) into ath5:gfp embryos that express the $g f p$ transgene under the regulation of the promoter for ath5, a zebrafish homolog of the Drosophila atonal gene (Masai et al., 2003). In these transgenic embryos, GFP is expressed by retinal cells, including the RGCs and their axons. When antisense MO was injected, RGC axons followed aberrant pathways within the retina in $47 \%$ of 42 cases after knockdown and in $0 \%$ of 40 control MO-injected retinas (Fig. 3A$C)$. RGC axons often extended away from the optic stalk, indicating a failure of guidance within the eye. Similar pathfinding defects by RGCs were also seen when the axons were labeled with MAb Zn5, which labels RGCs (Trevarrow et al., 1990) after injection of both $s d f-1 a$ and $c x c r 4 b$ MOs ( $40 \%$ of 20 retinas) (Fig. 4) or with antiacetylated $\alpha$-tubulin after injection of the $s d f-1 a$ (2-3 ng; $19 \%$ of 26 retinas) or cxcr $4 b$ (2-3 ng; $13 \%$ of 24 retinas) MO alone but not $s d f-1 b$ alone (2-3 ng; $0 \%$ of 14 retinas) (data not shown).

There were no obvious defects, other than pathfinding defects, in the retina of antisense morphant embryos. The effects of MO knockdown of SDF-1a/CXCR4b signaling appear to be direct rather than secondary to changes in specification or patterning of the retina or optic stalk. Neither knockdown of SDF-1a or CXCR4b, nor double knockdown of SDF-1a and CXCR4b, affected the overall architecture of the retina as seen with differential interference optics microscopy and histological sections of the eye $(n=6)$. Similarly, the optic stalk was unperturbed as indicated by pax $2 a(n=20)$ expression (Fig. 5$)$. Furthermore, the expression of EphB3 $(n=15)$ and ephrinB2 $(n=$ 18 ) in the retina (Fig. 5), expression of sema3a by the lens $(n=22$; data not shown), nor expression of netrin-1a $(n=24)$ in the optic stalk was perturbed in antisense morphants (Fig. 5). This is consistent with the hypothesis that the pathfinding defects seen in antisense morphants are not caused by changes in the expression patterns of molecules known to affect axonal outgrowth but are caused by decreased SDF-1a/CXCR4b signaling. 

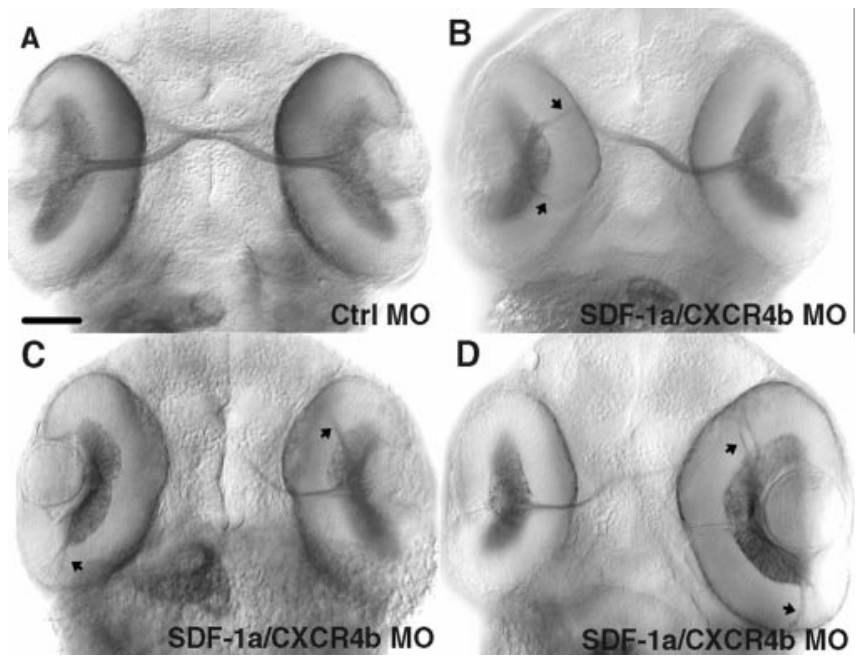

Figure 4. Retinal axons make pathfinding errors as a result of reduced SDF-1a/CXCR4b signaling after MO knockdown. All panels are ventral views of $48 \mathrm{hpf}$ embryos labeled with MAb $\mathrm{Zn} 5$, which stains both RGC cell bodies and axons. $A$, The optic nerve and RGCs labeled by MAb Zn5 in an embryo injected with control MO. B-D, RGC axons frequently projected aberrantly (arrows) in embryos injected with sdf-1a and $c x c r 4 b$ antisense MOs. In some cases (left-side retinas in $B$ and $C$, no optic nerve formed with all MAb $Z n 5^{+}$retinal axons projecting abnormally within the eye. Scale bar, $20 \mu \mathrm{m}$.

\section{Pathfinding by retinal axons is disrupted in $c x c r 4 b$ mutant odysseus embryos}

The odysseus (ody) mutant was first identified by a defect in migration by primordial germ cells caused by a putative loss-offunction mutation in the cxcr4b gene (Knaut et al., 2003). Because knockdown of SDF-1/CXCR4 signaling caused RGC axons to follow aberrant pathways in the retina, we examined axonal outgrowth by RGCs in $o d y^{t 26035}$ embryos. Indeed, retinal axons projected in erroneous directions to form prominent tracts within the retina of all eight mutants examined (Fig. 6). In many cases, axons extended away from the exit site of the retina. Interestingly, in some cases, projection errors were only seen in one of the two eyes in mutants much like that observed in SDF-1a/CXCR4b morphants (Fig. 4). As in the case of embryos with SDF-1/CXCR4 signaling knocked down, observation of the retina in mutants with differential interference contrast microscopy revealed no obvious defects (data not shown). This result strongly supports the knockdown result and demonstrates that SDF-1/CXCR4 signaling is important for correct pathfinding by RGC axons to the exit point of the retina. Furthermore, because RGCs project axons in both $s d f-1 a / c x c r 4 b$ antisense morphant embryos and in ody embryos, SDF-1/CXCR4 signaling appears not to be required for outgrowth of RGC axons but rather serves a pathfinding function for these axons.

Retinal axons are attracted by retinal cells misexpressing sdf-1 Errors by RGC axons after knockdown of SDF-1/CXCR4 signaling in wild-type embryos and in ody mutants demonstrate that signaling through this chemokine serves a pathfinding function but does not explain how SDF-1 guides RGC axons. The expression pattern of $s d f-1 a$ and $s d f-1 b$ by the optic stalk suggests that SDF-1 attracts RGC axons to the optic stalk. To test this hypothesis, pathfinding by RGC axons was examined in retinas that contained ectopic sources of SDF-1 to determine whether the axons were attracted by the ectopic sources. This was accomplished by transplanting cells from $h s p 70: s d f-1 b-g f p$ transgenic embryos into wild-type hosts at $4-5 \mathrm{hpf}$ and heat-inducing ex-

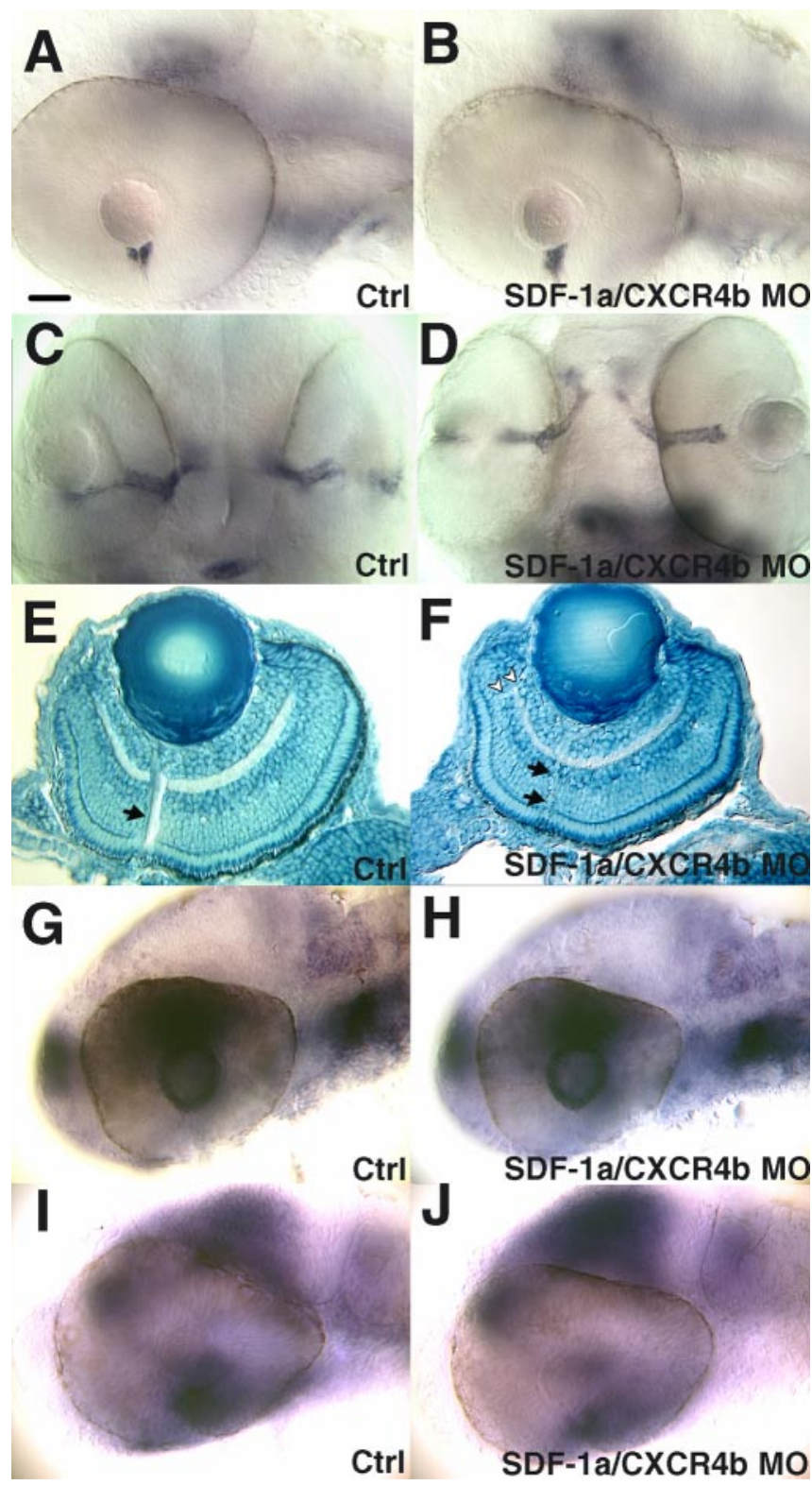

Figure 5. Perturbation of SDF-1/CXCR4 expression does not affect the patterning of the retina and optic stalk, nor does it change the expression pattern of other guidance molecules. $A$, Expression of netrin- $1 a$ by the head of the optic stalk adjacent to the eye is normal after heat induction as seen in a side view of a $48 \mathrm{hpf}$ control M0-injected embryo. Anterior is left; dorsal is up. $B$, Expression of netrin- $1 a$ is unperturbed after antisense SDF- $1 \mathrm{a}$ and antisense CXCR4b MO injection. $C$, Expression of pax2a by the optic stalk seen in a ventral view of a $48 \mathrm{hpf}$ embryo injected with control MO. D, Expression of pax2a is unperturbed after knockdown of SDF-1a/ CXCR4b. E, Sagittal section of the eye stained with methylene blue, showing the architecture of a $72 \mathrm{hpf}$ embryo after injection of control MOs. The arrow denotes the optic axons exiting the retina. F, Knockdown of SDF-1a and CXCR4b does not disturb the architecture of the eye. Note the thin bundle of optic axons exiting the retina (arrow) and the aberrant axon tract (arrowheads). $G$, Side view of a control M0 injected $32 \mathrm{hpf}$ embryo showing the expression of ephrinB2 $a$ by cells in the lens and the dorsal retina. $H$, Knockdown of SDF- $1 a$ and CXCR4b does not alter eprhinB2a expression. I, Side views of control or MO-injected embryos at 32 hpf showing ephB3 expression in the nasal region of the retina as well as the tectum. The apparent expression seen in the dorsal retina in / and in $J$ is actually expression by the brain just medial to the eye. J, ephB3 expression was not disturbed by SDF-1a/CXCR4b knockdown. Scale bar, $20 \mu \mathrm{m}$.

pression of the SDF-1b GFP at 28, 32, and $36 \mathrm{hpf}$ and assayed for RGC axons with anti-acetylated $\alpha$-tubulin at $40 \mathrm{hpf}$. Under these conditions, embryos were mosaics with transgenic cells scattered randomly within the wild-type host. Control embryos were 

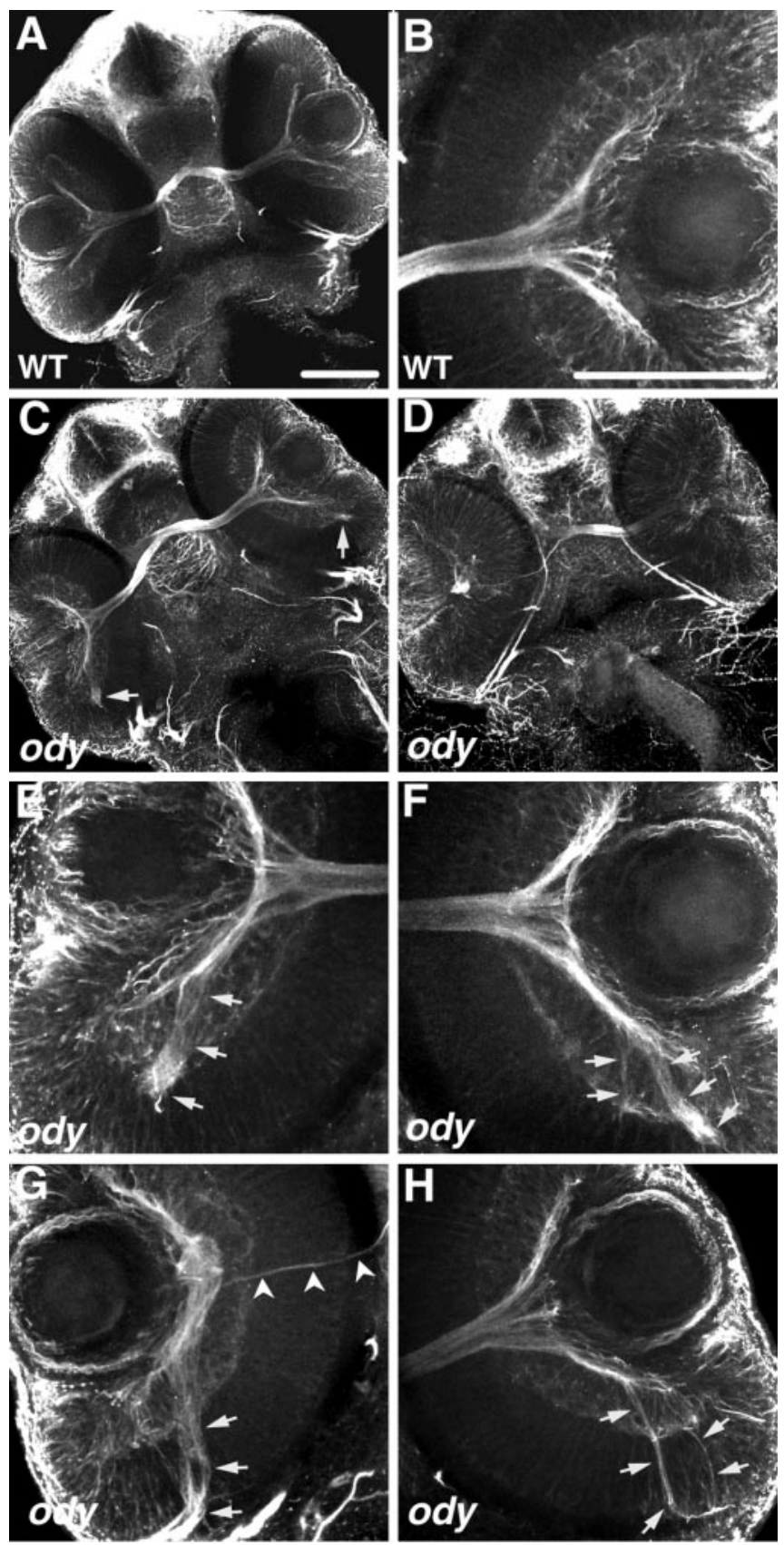

Figure 6. The $c x c r 4 b$ mutant, odysseus (ody), displays pathfinding defects by RGC axons within the retina. All panels are confocal images of ventrally mounted $48 \mathrm{hpf}$ embryos labeled with anti-acetylated $\alpha$-tubulin. $A, B$, Retinas and optic nerves in a wild-type embryo $(A)$ and close-up view of the retina and adjacent optic nerve ( $B)$. $C-H, R G C$ axons in ody embryos frequently extend in aberrant directions within the retina (arrows). Retinas of embryo in $C$ are shown under higher magnification in $E$ and $F$; those of embryo in $D$ are shown in higher magnification in $G$ and $H$. Arrowheads in $G$ indicate the axons projecting normally, exiting at the optic nerve head, whereas the majority of RGC axons project posteriorly (arrow). Scale bar, $20 \mu \mathrm{m}$.

transplanted with cells from $h s p 70: g f p^{k 6}$ embryos and heat induced. In control embryos, the pathways of RGC axons were normal in all retinas $(n=33)$ that contained GFP-expressing cells. However, RGC axons were found in aberrant positions along or within $2 \mu \mathrm{m}$ from SDF-1b GFP-expressing cells within the retina in $40 \%$ of retinas that contained GFP-expressing cells $(n=25)$ in experimental embryos (Fig. 7). The correlation of deviant RGC axons and SDF-1b GFP-expressing cells suggests that SDF-1 acts as a chemoattractant for RGC axons and that
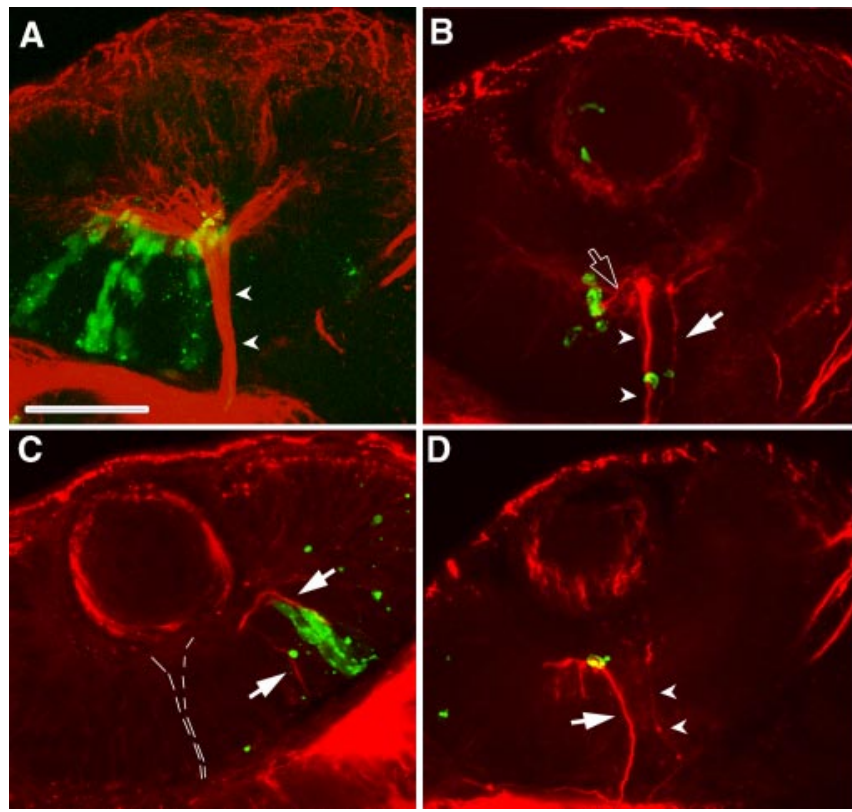

Figure 7. Misdirected RGC axons are located along retinal cells ectopically expressing SDF1b. All panels are confocal images of ventrally mounted $48 \mathrm{hpf}$ wild-type embryos containing transplanted cells from $h s p: g f p$ or $h s p: s d f-1 b g f p$ embryos. Host embryos were heat induced so that the donor cells expressed GFP or SDF-1b-GFP. Axons are labeled with anti-acetylated $\alpha$-tubulin (red) and transplanted cells by GFP (green). A, GFP retinal cells do not affect pathfinding by $\mathrm{RGC}$ axons. Arrowheads mark the optic nerve. $B, C, \mathrm{RGC}$ axons appear to extend along retinal cells expressing SDF-1b-GFP. B, Two misdirected bundles of RGC axons correspond to cells ectopically expressing SDF-1b: one projects away from the optic nerve head in a ventral and anterior direction (open arrow) and another (arrow) in parallel to the normal optic nerve (arrowheads). C, Two aberrant bundles of RGC axons (arrows) extend away from the RGC layer toward SDF-1b-expressing cells. The optic axons exiting the retina through the optic stalk (dashed lines) are not visible at this focal plane. Scale bar, $20 \mu \mathrm{m}$.

SDF-1b and perhaps SDF-1a secreted by the initial portion of the optic stalk serves to attract RGC axons to the head of the optic nerve to guide them out of the retina.

\section{Discussion}

\section{Axonal guidance by SDF-1/CXCR4 signaling}

Both knockdown of SDF-1/CXCR4 signaling and mutations in $c x c r 4 b$ adversely affected the pathways followed by RGC axons. Furthermore, the pathways followed by RGC axons were aberrant in retinas ectopically expressing SDF-1, and the pathways were along or in the vicinity of ectopically expressing cells. The simplest interpretation of our results is that SDF-1s may serve as a chemoattractant for RGC growth cones. This interpretation can account for the strikingly similar retinal axon phenotypes observed in shh mutants and knock-outs (Schauerte et al., 1998; Dakubo et al., 2003). In these embryos, a loss of Shh from the retinal ganglion cells leads to defects in the differentiation of the optic stalk, which could lead to a loss of stalk-derived SDF-1 and subsequent retinal axon defects. The idea that SDF-1 is a chemoattractant for retinal axons is supported by the fact that SDF-1 is a chemoattractant for cells in the immune system and migrating cerebellar and dentate granule cells (Cho and Miller, 2002). Consonant with this idea, other molecules such as semaphorins, netrins, slits, and ephrins regulate both cell migration and growth cone guidance (Park et al., 2002). Furthermore, SDF-1/CXCR4 signaling can affect turning by growth cones in vitro and pathfinding by cutaneous sensory axons in the spinal cord (Xiang et al., 2002; Chalasani et al., 2003a). Counter to this hypothesis, in vitro SDF-1 apparently exerts no direct chemoattractive effect on 
chick RGC axons (Chalasani et al., 2003a). This might be because of species differences and/or in vitro versus in vivo differences or differences in the context or state of the growth cones. In fact, SDF- $1 \alpha$ has been reported to both induce apoptosis and promote survival or proliferation of neurons (Cho and Miller, 2002), suggesting that context may influence chemokine action. Finally, the response of growth cones to guidance molecules such as Netrin and Semaphorins can vary depending on the status of intracellular messengers $\mathrm{Ca}^{2+}$, cAMP, and cGMP (Song and Poo, 1999). Thus, the action of SDF-1 can vary, depending on the context, and could potentially explain the disparity in the in vitro and in vivo results.

A second possible mechanism for SDF-1/CXCR4 effects on pathfinding is the regulation of the action of other guidance molecules by SDF-1/CXCR4. For example, SDF-1/CXCR4 may potentiate the effects of Netrin/deleted in colorectal cancer (DCC). Netrin-1 is expressed by the head of the optic stalk in a similar manner to the SDF-1s and can stimulate neurite outgrowth from RGCs (Deiner et al., 1997; Lauderdale et al., 1997). Furthermore, in Netrin-1 and DCC knock-outs, RGC axons extend normally to the optic disc but failed to exit the eye and instead extended aberrantly within the eye. This suggests that Netrin-1/DCC signaling is not necessary for guidance to the optic disc and thus does not mediate an essential chemoattractive function but is required for exiting the eye. This contrasts with RGC axons in SDF-1a/CXCR4b knock-down embryos that failed to extend to the optic stalk and instead extended aberrantly within the eye. Because the Netrin/DCC and SDF-1/CXCR4 loss-of-function phenotypes differ from each other, it is unlikely that SDF-1/ CXCR4 simply acts to potentiate the action of Netrin/DCC on RGC axons.

SDF-1/CXCR4 can interact with various axonal repellants, and this could potentially account for the pathfinding errors made by RGC axons in our experiments. Slit- 1 and Slit- 2 inhibit RGC axons (Erskine et al., 2000; Niclou et al., 2000; Ringstedt et al., 2000; Plump et al., 2002), and application of SDF-1 in vitro reduces the repulsive action of Slit-2 (Chalasani et al., 2003a). Slit-1a and Slit-1b are both expressed in the zebrafish retina and Slit- 3 by the lens, but Slit- 2 is not expressed in the retina (Yeo et al., 2001; Hutson and Chien, 2002; Hutson et al., 2003). Thus, silencing of repulsive actions of Slit-1 and/or Slit-3 by SDF-1/ CXCR4 could participate in guiding RGC axons within the zebrafish retina. SDF-1/CXCR40-mediated silencing of repulsive molecules may also play a role in guidance of RGC axons within the optic stalk and chiasm. Slit-2 is expressed by the optic stalk in both mammals and zebrafish (Niclou et al., 2000; Hutson and Chien, 2002), and RGC axons make errors in and near the optic chiasm in double mouse knock-outs of Slit-1 and Slit-2 (Plump et al., 2002) and in zebrafish mutant for robo2 that encodes a Slit receptor (Fricke et al., 2001). Thus, previous exposure of RGC growth cones to SDF-1s in the optic stalk could potentially reduce the repulsive action of Slit-2 to allow RGC axons to extend through the optic stalk and chiasm.

SDF-1, Netrin-1, and Slit-2 all affect RGC axon extension. How might these molecules act within the visual pathway to guide RGC axons? The SDF-1s and Netrin-1a are expressed by the head of the optic stalk in zebrafish. SDF-1 proteins are small proteins that may diffuse farther than the larger Netrin-1, which is thought to act locally at the optic disc (Deiner et al., 1997). We propose that the SDF-1s are distributed as a gradient within the retina and act as a chemoattractant to guide RGC axons within the retina to the optic stalk. Once at the head of the optic stalk, the growth cones interact with Netrin-1, which guides them out of the eye into the optic stalk. Furthermore, within the optic stalk and chiasm, previous exposure of RGC growth cones to SDF-1s in the head of the optic stalk then reduces the repulsive effects of Slit-2 to allow them to traverse through the stalk and chiasm. Finally, SDF-1/CXCR4 signaling also mediates a trophic activity on RGCs (Chalasani et al., 2003b). Thus, SDF-1/CXCR4 signaling may be active both for trophic support of neurons and guidance of their axons.

\section{References}

Chalasani SH, Sabelko KA, Sunshine MJ, Littman DR, Raper JA (2003a) A chemokine, SDF-1, reduces the effectiveness of multiple axonal repellents and is required for normal axon pathfinding. J Neurosci 23:1360-1371.

Chalasani SH, Baribaud F, Coughlan CM, Sunshine MJ, Lee VM, Doms RW, Littman DR, Raper JA (2003b) The chemokine stromal cell-derived factor-1 promotes the survival of embryonic retinal ganglion cells. J Neurosci 23:4601-4612.

Cho C, Miller RJ (2002) Chemokine receptors and neural function. J Neurovirol 8:573-584.

Chong SW, Emelyanov A, Gong Z, Korzh V (2001) Expression pattern of two zebrafish genes, cxcr4a and cxcr4b. Mech Dev 109:347-354.

Dakubo GD, Wang YP, Mazerolle C, Campsall K, McMahon AP, Wallace VA (2003) Retinal ganglion cell-derived sonic hedgehog signaling is required for optic disc and stalk neuroepithelial cell development. Development 130:2967-2980.

David NB, Sapede D, Saint-Etienne L, Thisse C, Thisse B, Dambly-Chaudiere C, Rosa FM, Ghysen A (2002) Molecular basis of cell migration in the fish lateral line: role of the chemokine receptor CXCR4 and of its ligand, SDF1. Proc Natl Acad Sci USA 99:16297-16302.

Deiner MS, Kennedy TE, Fazeli A, Serafini T, Tessier-Lavigne M, Sretavan DW (1997) Netrin-1 and DCC mediate axon guidance locally at the optic disc: loss of function leads to optic nerve hypoplasia. Neuron 19:575-589.

Doitsidou M, Reichman-Fried M, Stebler J, Koprunner M, Dorries J, Meyer D, Esguerra CV, Leung T, Raz E (2002) Guidance of primordial germ cell migration by the chemokine SDF-1. Cell 111:647-659.

Erskine L, Williams SE, Brose K, Kidd T, Rachel RA, Goodman CS, TessierLavigne M, Mason CA (2000) Retinal ganglion cell axon guidance in the mouse optic chiasm: expression and function of robos and slits. J Neurosci 20:4975-4982.

Fricke C, Lee JS, Geiger-Rudolph S, Bonhoeffer F, Chien CB (2001) Astray, a zebrafish roundabout homolog required for retinal axon guidance. Science 292:507-510.

Halloran MC, Sato-Maeda M, Warren JT, Su F, Lele Z, Krone PH, Kuwada JY, Shoji W (2000) Laser-induced gene expression in specific cells of transgenic zebrafish. Development 127:1953-1960.

Ho RK, Kane DA (1990) Cell-autonomous action of zebrafish spt-1 mutation in specific mesodermal precursors. Nature 348:728-730.

Hutson LD, Chien CB (2002) Pathfinding and error correction by retinal axons: the role of astray/robo2. Neuron 33:205-217.

Hutson LD, Jurynec MJ, Yeo SY, Okamoto H, Chien CB (2003) Two divergent slit1 genes in zebrafish. Dev Dyn 228:358-369.

Kimmel CB, Ballard WW, Kimmel SR, Ullmann B, Schilling TF (1995) Stages of embryonic development of the zebrafish. Dev Dyn 203:253-310.

Knaut H, Werz C, Geisler R, Nusslein-Volhard C (2003) A zebrafish homologue of the chemokine receptor Cxcr4 is a germ-cell guidance receptor. Nature 421:279-282.

Lauderdale JD, Davis NM, Kuwada JY (1997) Axon tracts correlate with netrin-1a expression in the zebrafish embryo. Mol Cell Neurosci 9:293-313.

Li Q, Shirabe K, Kuwada JY (2004) Chemokine signaling regulates sensory cell migration in zebrafish. Dev Biol 269:123-136.

Masai I, Lele Z, Yamaguchi M, Komori A, Nakata A, Nishiwaki Y, Wada H, Tanaka H, Nojima Y, Hammerschmidt M, Wilson SW, Okamoto H (2003) N-cadherin mediates retinal lamination, maintenance of forebrain compartments and patterning of retinal neurites. Development 130:2479-2494.

Nasevicius A, Ekker SC (2000) Effective targeted gene "knockdown" in zebrafish. Nat Genet 26:216-220.

Niclou SP, Jia L, Raper JA (2000) Slit2 is a repellent for retinal ganglion cell axons. J Neurosci 20:4962-4974.

Park HT, Wu J, Rao Y (2002) Molecular control of neuronal migration. BioEssays 24:821-827. 
Plump AS, Erskine L, Sabatier C, Brose K, Epstein CJ, Goodman CS, Mason CA, Tessier-Lavigne M (2002) Slit1 and Slit2 cooperate to prevent premature midline crossing of retinal axons in the mouse visual system. Neuron 33:219-232.

Postlethwait JH, Woods IG, Ngo-Hazelett P, Yan YL, Kelly PD, Chu F, Huang H, Hill-Force A, Talbot WS (2000) Zebrafish comparative genomics and the origins of vertebrate chromosomes. Genome Res 10:1890-1902.

Ringstedt T, Braisted JE, Brose K, Kidd T, Goodman C, Tessier-Lavigne M, O'Leary DD (2000) Slit inhibition of retinal axon growth and its role in retinal axon pathfinding and innervation patterns in the diencephalon. J Neurosci 20:4983-4991.

Rossi D, Zlotnik A (2000) The biology of chemokines and their receptors. Annu Rev Immunol 18:217-242.

Schauerte HE, van Eeden FJ, Fricke C, Odenthal J, Strahle U, Haffter P (1998) Sonic hedgehog is not required for the induction of medial floor plate cells in the zebrafish. Development 125:2983-2993.

Schulte-Merker S, Ho RK, Herrmann BG, Nusslein-Volhard C (1992) The protein product of the zebrafish homologue of the mouse $\mathrm{T}$ gene is expressed in nuclei of the germ ring and the notochord of the early embryo. Development 116:1021-1032.
Song HJ, Poo MM (1999) Signal transduction underlying growth cone guidance by diffusible factors. Curr Opin Neurobiol 9:355-363.

Tran PB, Miller RJ (2003) Chemokine receptors in the brain: a developing story. J Comp Neurol 457:1-6.

Trevarrow B, Marks DL, Kimmel CB (1990) Organization of hindbrain segments in the zebrafish embryo. Neuron 4:669-679.

Westerfield M (1995) The zebrafish book: a guide for the laboratory use of zebrafish (Brachydanio rerio). In: The zebrafish book, Ed 2, pp 8.1-1.22. Eugene, OR: University of Oregon.

Xiang Y, Li Y, Zhang Z, Cui K, Wang S, Yuan XB, Wu CP, Poo MM, Duan S (2002) Nerve growth cone guidance mediated by G protein-coupled receptors. Nat Neurosci 5:843-848.

Xiao T, Shoji W, Zhou W, Su F, Kuwada JY (2003) Transmembrane sema4E guides branchiomotor axons to their targets in zebrafish. J Neurosci 23:4190-4198.

Yeo SY, Little MH, Yamada T, Miyashita T, Halloran MC, Kuwada JY, Huh TL, Okamoto H (2001) Overexpression of a slit homologue impairs convergent extension of the mesoderm and causes cyclopia in embryonic zebrafish. Dev Biol 230:1-17. 